\title{
The Natural History of the Normal First Stage of Labor
}

\author{
Jun Zhang, PhD, MD, James Troendle, PhD, Rafael Mikolajczyk, MD, MSc, Rajeshwari Sundaram, PhD, \\ Julie Beaver, $M S$, and William Fraser, MD, MS
}

OBJECTIVE: To examine labor patterns in a large population and to explore an alternative approach for diagnosing abnormal labor progression.

METHODS: Data from the National Collaborative Perinatal Project were used. A total of 26,838 parturients were selected who had a singleton term gestation, spontaneous onset of labor, vertex presentation, and a normal perinatal outcome. A repeated-measures analysis was used to construct average labor curves by parity. An interval-censored regression was used to estimate duration of labor stratified by cervical dilation at admission and centimeter by centimeter.

RESULTS: The median time needed to progress from one centimeter to the next became shorter as labor advanced (eg, from 1.2 hours at $3-4 \mathrm{~cm}$ to 0.4 hours at $7-8 \mathrm{~cm}$ in nulliparas). Nulliparous women had the longest and most gradual labor curve; multiparous women of different parities had very similar curves. Nulliparas may start the active phase after $5 \mathrm{~cm}$ of cervical dilation and may not necessarily have a clear active phase characterized by precipitous dilation. The deceleration phase in the late active phase of labor may be an artifact in many cases.

$\overline{\text { From the Division of Epidemiology, Statistics and Prevention Research, Eunice }}$ Kennedy Shriver National Institute of Child Health and Human Development, National Institutes of Health, Bethesda, Maryland; the Department of Clinical Epidemiology, Bremen Institute for Prevention Research and Social Medicine, Bremen University, Bremen, Germany; and the Department of Obstetrics and Gynecology, University of Montreal, Montreal, Quebec, Canada.

Supported by the Intramural Program of the Eunice Kennedy Shriver National Institute of Child Health and Human Development, National Institutes of Health.

The authors thank Dr. Ann Trumble for preparing the database.

Presented at the Society for Maternal-Fetal Medicine 29th Annual Meeting, January 26-31, 2009, San Diego, California.

Corresponding author: Jun Zhang, MD, PhD, Epidemiology Branch, NICHD, National Institutes of Health, Building 6100, Room 7B03, Bethesda, MD 20892; e-mail: zhangj@mail.nih.gov.

Financial Disclosure

Dr. Fraser receives salary support from the Canadian Institutes of Health Research through a Canada Research Chair. The other authors did not report any potential conflicts of interest.

(c) 2010 by The American College of Obstetricians and Gynecologists. Published by Lippincott Williams \& Wilkins.

ISSN: 0029-7844/10
CONCLUSION: The active phase of labor may not start until $5 \mathrm{~cm}$ of cervical dilation in multiparas and even later in nulliparas. A 2-hour threshold for diagnosing labor arrest may be too short before $6 \mathrm{~cm}$ of dilation, whereas a 4-hour limit may be too long after $6 \mathrm{~cm}$. Given that cervical dilation accelerates as labor advances, a graduated approach based on levels of cervical dilation to diagnose labor protraction and arrest is proposed.

(Obstet Gynecol 2010;115:705-10)

\section{LEVEL OF EVIDENCE: III}

$T^{\mathrm{h}}$ he escalating rate of cesarean delivery worldwide has caused serious concerns in the obstetric community and the public. ${ }^{1,2}$ Causes for the high rate are complex. ${ }^{3}$ Currently, there is a lack of convincing and effective strategies to reverse the trend.

Our inability to reduce the cesarean rate may be attributable in part to the incomplete understanding of a normal labor process, particularly in the first stage of labor. However, owing to various interventions in contemporary obstetric practice, a natural process of labor progression no longer can be studied in a large population. The high rate of elective and intrapartum cesarean delivery may further cause serious selection bias. Thus, we sought to study the first stage of labor using data from the National Collaborative Perinatal Project, a large, multicenter, prospective, observational study conducted between 1959 and 1966. ${ }^{4}$ Because obstetric interventions in the first stage of labor were less common in that era, these data provide a unique opportunity to observe what may be closest to the natural process of labor in a large population. The objective of our study was to examine labor patterns in a large population and to explore an alternative approach for diagnosing abnormal labor progression.

\section{MATERIALS AND METHODS}

The National Collaborative Perinatal Project was designed to study factors during pregnancy and early childhood that affect child neurodevelopment and 
diseases such as cerebral palsy. Abnormal progress of labor was one of the factors of interest. Women who sought prenatal care at 12 hospitals in the United States were recruited prospectively from 1959 to 1965. ${ }^{4}$ At entry, detailed demographic, socioeconomic, and behavioral data were collected through in-person interviews. Medical history, physical examination, and blood sample also were obtained. In the ensuing prenatal visits, women were interviewed repeatedly and physical findings were recorded. When the woman was admitted to the hospital for labor and delivery, her physical status was reevaluated and all labor and delivery events were recorded in detail by a trained observer. However, no particular labor protocol was used, and the number and timing of pelvic exams were not standardized. A summary of the labor and delivery was completed by the obstetrician responsible for the patient's care. ${ }^{5}$ Children were observed up to 7 years of age. A comprehensive battery of physical exams and psychological and neurodevelopmental tests were conducted. ${ }^{6}$

The current study uses information on maternal baseline characteristics, physical findings at admission to labor and delivery, repeated measures of cervical dilation, and delivery information. Friedman et $\mathrm{al}^{5}$ conducted a detailed validation study on errors of the labor data in the National Collaborative Perinatal Project. A sample of 667 gravidas was selected for whom there were suspicious labor data entry errors on cervical dilation, fetal station, or date and time of the exams because the labor progress was unusually rapid or dilatation regressed. A total of 17,916 data items from these cases were verified against hardcopy records. The authors found that the overall error rate was about $1 \%$ with the error rate of $0.96 \%, 0.87 \%$, $1.11 \%$ and $1.24 \%$ for date, time, cervical dilation and fetal station, respectively. Conversely, they selected 1,764 dilation values, 1,925 station values, 1,901 time values and 1,901 date values that were not suspected of being problematic in any way. The error rates were $1.25 \%, 1.19 \%, 1.26 \%$ and $0 \%$, respectively. Therefore, it was concluded that the National Collaborative Perinatal Project labor data were reasonably complete and accurate. During our own data preparation for the current analysis, we identified a problem related to time. In one hospital, the clock in the delivery room was not correctly adjusted for daylight savings time, which resulted in discrepancies in time between labor and delivery records. A total of 2,718 records were affected by this error and, therefore, fixed by adding 1 hour to the delivery time in these cases.

A total of 54,304 singleton pregnancies who were delivered at 20 weeks or later were included in the study. We restricted our analysis to term pregnancies (37-42 weeks, $\mathrm{n}=40,973$ ) with a spontaneous onset of labor $(n=36,402)$. We also restricted to pregnancies with vertex fetal presentation at admission (excluding face, brow, or chin position, $n=35,318)$. Further excluded were cases of placenta previa, severe hypertension in pregnancy, cord prolapse, and uterine rupture $(\mathrm{n}=34,250)$. To examine the labor pattern in parturients with normal perinatal outcomes, we restricted our analysis to births of neonates with 5-minute Apgar scores of at least $7(n=30,872)$. Finally, we excluded women who had fewer than two values of cervical dilation in the first stage $(n=27,801)$ or never reached $10 \mathrm{~cm}$, leaving 26,838 women for analysis.

Rectal exams to measure cervical dilation were performed quite commonly in that era. Among 262,262 pelvic exams in the National Collaborative Perinatal Project data, 45\% were vaginal, $45 \%$ were rectal, and 10\% were unknown. We compared two types of measurements in terms of degree of error and effect on labor curves and found no clinically significant differences (results not shown). Thus, we used both types of measurements in our analysis.

We first grouped the participants according to their parity $(0,1,2+)$ and compared baseline characteristics. We used the $\chi^{2}$ test for categorical variables, analysis of variance for continuous variables with normal distribution, and nonparametric rank-sum test for continuous variables with skewed distribution to examine statistical significance. We then used a repeated-measures analysis ${ }^{7}$ with an eighth-degree polynomial model to construct average labor curves by parity. In this analysis, the starting point was set at the first time when the dilation reached $10 \mathrm{~cm}($ time $=0)$ and the time was calculated backwards (eg, 60 minutes before the complete dilation, -60 minutes). After the labor curve models had been computed, the $\mathrm{X}$ axis (time) was reverted to a positive value, ie, instead of being $-12 \rightarrow 0$ hours, it became $0 \rightarrow 12$ hours.

Labor progression data are interval censored, eg, one only knows that the cervix dilated from 4 to $5 \mathrm{~cm}$ between two pelvic exams but does not know exactly when it happened. Thus, we used an interval censored regression ${ }^{8}$ to estimate the distribution of times for progression from one integer centimeter of dilation to the next (called traverse time) with an assumption that the labor data are log-normally distributed. ${ }^{9}$ The median and 95th percentiles were calculated. A total of $6.6 \%$ of women with parity of two or more contributed more than one pregnancy data to that group $(\mathrm{P} 2+)$. We therefore used a parametric frailty model to account for the repeated measures in these women. ${ }^{10}$ Because multiparous women tended to be 
admitted at a more advanced stage labor than nulliparous women, many multiparous women did not have information on cervical dilation before $3.5 \mathrm{~cm}$. Therefore, the labor curves and traverse time for multiparous women started at $3.5 \mathrm{~cm}$ (or $4 \mathrm{~cm}$ ) rather than at $3 \mathrm{~cm}$ as for nulliparous women.

Finally, to address the clinical experience where a woman is first observed at a given dilation and then measured periodically, we calculated cumulative duration of labor from admission to any given dilation up to the first $10 \mathrm{~cm}$ in nulliparas. The same interval censored regression approach was used. We provide the estimates according to the dilation at admission $(2.0$ or $2.5 \mathrm{~cm}, 3.0$ or $3.5 \mathrm{~cm}, 4.0$ or $4.5 \mathrm{~cm}, 5.0$ or $5.5 \mathrm{~cm}$ ) because women admitted at different dilation levels may have different patterns of labor progression. All statistical analyses were performed using SAS 9.1 (SAS Institute Inc., Cary, NC; PROC MIXED for the repeated-measures analysis,
PROC LIFEREG for interval censored regression and PROC NLMIXED for interval censored regression with repeated measures). Because the National Collaborative Perinatal Project data are publicly available, our study was exempted from institutional review board review.

\section{RESULTS}

In National Collaborative Perinatal Project, the overall rate of cesarean delivery was $5.6 \%$; in women who had trial of labor, the cesarean rate was $2.6 \%$ and the induction rate was $7.1 \%$. Table 1 presents the maternal characteristics of the selected study population by parity. Approximately half of the women were white, 40\% were African American, and 8\% were other, mainly Hispanic. The socioeconomic status index (combination of education, occupation and household income) suggests that the majority of women were between the 20th and 80th percentiles based on 1960 U.S.

Table 1. Characteristics of the Parturients by Parity, National Collaborative Perinatal Project, 1959-1966

\begin{tabular}{|c|c|c|c|}
\hline & $\begin{array}{c}\text { Parity 0 } \\
(n=8,690)\end{array}$ & $\begin{array}{c}\text { Parity } 1 \\
(n=6,373)\end{array}$ & $\begin{array}{c}\text { Parity } 2+ \\
(n=11,765)\end{array}$ \\
\hline \multicolumn{4}{|l|}{ Maternal race } \\
\hline White & 53 & 55 & 45 \\
\hline African American & 39 & 38 & 48 \\
\hline Other & 8 & 7 & 7 \\
\hline \multicolumn{4}{|l|}{ Socioeconomic status index (percentile) } \\
\hline Less than 20th & 8 & 5 & 7 \\
\hline 20th-39th & 24 & 26 & 34 \\
\hline 40th-59th & 28 & 32 & 33 \\
\hline 60th-79th & 23 & 24 & 19 \\
\hline 80th or higher & 17 & 13 & 7 \\
\hline Maternal age $(y)$ & $20.3 \pm 3.9$ & $22.7 \pm 4.4$ & $27.4 \pm 5.5$ \\
\hline Prepregnancy BMI (kg/m²) & $21.5 \pm 3.2$ & $22.2 \pm 3.8$ & $23.5 \pm 4.5$ \\
\hline Weight gain $(\mathrm{kg})$ & $10.9 \pm 4.5$ & $10.3 \pm 4.4$ & $10.3 \pm 4.9$ \\
\hline BMI at delivery $\left(\mathrm{kg} / \mathrm{m}^{2}\right)$ & $25.4 \pm 3.5$ & $25.9 \pm 3.9$ & $27.1 \pm 4.5$ \\
\hline Cervical dilation at admission $(\mathrm{cm})$ & $3(1,6)$ & $3.5(2,7)$ & $3.5(1.5,6.5)$ \\
\hline Station at admission & $0(-2,1)$ & $0(-2,1)$ & $-1(-3,1)$ \\
\hline Effacement at admission (\%) & $85(50,100)$ & $80(45,100)$ & $75(30,100)$ \\
\hline Rupture of the membranes at admission & 29 & 26 & 27 \\
\hline Oxytocin for augmentation & 20 & 12 & 12 \\
\hline Regional analgesia (caudal, sacral, epidural, peridural) & 8 & 11 & 8 \\
\hline Total number of pelvic exams in first stage & $6(3,11)$ & $5(2,9)$ & $5(2,9)$ \\
\hline \multicolumn{4}{|l|}{ Forceps delivery } \\
\hline None & 27 & 55 & 76 \\
\hline Outlet & 33 & 17 & 9 \\
\hline Low & 27 & 16 & 8 \\
\hline Mid/high & 13 & 12 & 7 \\
\hline Cesarean delivery at second stage & 0.24 & 0.02 & 0.05 \\
\hline Gestational age at delivery (wk) & $39.8 \pm 1.4$ & $39.8 \pm 1.4$ & $39.8 \pm 1.4$ \\
\hline Birth weight (g) & $3,185 \pm 434$ & $3,259 \pm 443$ & $3,301 \pm 483$ \\
\hline White & $3,290 \pm 431$ & $3,337 \pm 446$ & $3,390 \pm 487$ \\
\hline African American & $3,060 \pm 409$ & $3,155 \pm 424$ & $3,220 \pm 467$ \\
\hline Other & $3,118 \pm 403$ & $3,225 \pm 402$ & $3,295 \pm 477$ \\
\hline
\end{tabular}

BMI, body mass index.

Data are $\%$, mean \pm standard deviation, or median (10th, 90th percentile).

All differences among the three groups were statistically significant at $P<.01$ except for gestational age at delivery, where $P=.02$. 
census. $^{11}$ The mean maternal age was 20,23 , and 27 years for parity $0,1,2+$, respectively. Women were lean; gained $10 \mathrm{~kg}$, on average, during pregnancy.

The median cervical dilation at admission was 3 $\mathrm{cm}$ for nulliparas and $3.5 \mathrm{~cm}$ for multiparas. 20\% nulliparas and $12 \%$ multiparas received oxytocin for augmentation. 8 - 11\% of women used regional analgesia for labor pain (approximately half used caudal and sacral analgesia and half used epidural and peridural analgesia). Virtually all of them were delivered vaginally (cesarean deliveries at the first stage were excluded). The mean gestational age was 39.8 weeks for both nulliparas and multiparas. The mean birth weight increased with parity in all races.

Figure 1 depicts the average labor curves for the three parity groups. Nulliparous women had the longest and most gradual labor curve; multiparous women of different parities had very similar curves. The division between latent and active phases was more obvious in multiparas than in nulliparas. The inflection point appeared to have occurred earlier in parity $2+($ at $5 \mathrm{~cm})$ than in parity $1($ at $5.5 \mathrm{~cm})$. The nulliparous curve did not have a clear inflection point.

Table 2 provides a more detailed analysis on labor progression. The median time needed to progress from one centimeter to the next became shorter as the labor advanced. The 95th percentiles suggest that, in nulliparous women, labor may be slow before $6 \mathrm{~cm}$ but that it still can result in a vaginal delivery with a normal perinatal outcome.

Table 3 presents the median and 95th percentiles of the duration of labor in nulliparous women whose first cervical examination was at $2,3,4$, or $5 \mathrm{~cm}$ of dilation, respectively. For example, if a nulliparous woman with

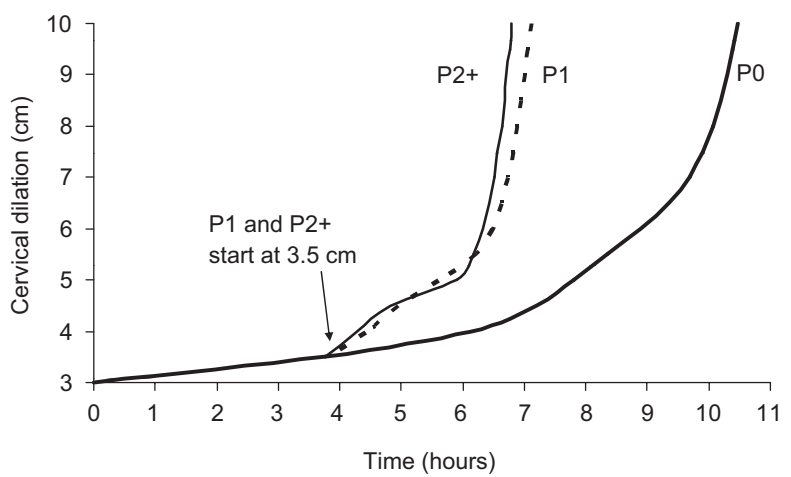

Fig. 1. The average labor curves by parity in women with singleton term pregnancies with spontaneous onset of labor and vertex presentation who completed the first stage of labor and whose newborns had 5-minute Apgar scores of at least 7. The National Collaborative Perinatal Project, 19591966. P0, nulliparas; P1, parity 1; P2 +, parity 2 or higher. Zhang. The First Stage of Labor. Obstet Gynecol 2010.
Table 2. Duration of Labor (in Hours) by Parity, National Collaborative Perinatal Project, 1959-1966

\begin{tabular}{llll}
\hline $\begin{array}{l}\text { Cervical } \\
\text { Dilation }(\mathbf{c m})\end{array}$ & Parity 0 & Parity $\mathbf{1}$ & Parity 2+ \\
\hline From 3 to 4 & $1.2(6.6)$ & & \\
From 4 to 5 & $0.9(4.5)$ & $0.7(3.3)$ & $0.7(3.5)$ \\
From 5 to 6 & $0.6(2.6)$ & $0.4(1.6)$ & $0.4(1.6)$ \\
From 6 to 7 & $0.5(1.8)$ & $0.4(1.2)$ & $0.3(1.2)$ \\
From 7 to 8 & $0.4(1.4)$ & $0.3(0.8)$ & $0.3(0.7)$ \\
From 8 to 9 & $0.4(1.3)$ & $0.3(0.7)$ & $0.2(0.6)$ \\
From 9 to 10 & $0.4(1.2)$ & $0.2(0.5)$ & $0.2(0.5)$ \\
From 4 to 10 & $3.7(16.7)$ & $2.4(13.8)$ & $2.2(14.2)$ \\
\hline
\end{tabular}

Data are median (95th percentile).

spontaneous onset of labor was admitted at $3 \mathrm{~cm}$, the median time from admission to $6 \mathrm{~cm}$ was approximately 2.7 hours and the 95th percentile was 12.3 hours. If a woman was admitted at $4 \mathrm{~cm}$, her median and 95th percentile of the duration of labor from admission to 6 $\mathrm{cm}$ were approximately 1.4 and 7.8 hours, respectively.

\section{DISCUSSION}

Labor progression was first studied systematically and extensively by Dr. Friedman for four decades (from the 1950 s to the 1980 s). A number of his highly influential articles and books established the landscape of this field. ${ }^{5,12-14}$ Perhaps because labor is often considered unpredictable and the methodology required to study labor is complex, the pace of research on this topic has been painstakingly slow. Only in more recent years has it regained some traction as cesarean delivery became pandemic. Unfortunately, because a large proportion of pregnancies now are delivered by cesarean, either before or during labor, selection bias (ie, who is left to have a vaginal delivery?) can have a serious effect on findings in any contemporary studies. The high frequency of obstetric intervention (eg, induction of labor) further complicates the data, which makes a natural history study difficult. It thus becomes more challenging to conduct statistical analyses that will yield unbiased results. We, therefore, based our analysis on the old National Collaborative Perinatal Project cohort where cesarean delivery was uncommon and the rate of labor induction relatively low. We describe the labor progression in the first stage only, as forceps were frequently used in the second stage of labor, particularly in nulliparas (33\%, 27\%, and 13\% for outlet, low, and mid/high forceps, respectively), which could have affected the course of the second stage.

One of the interesting findings of our study is that the inflection point on the average labor curves of the 
Table 3. Duration of Labor (in Hours) in Nulliparas Based on Cervical Dilation at Admission, National Collaborative Perinatal Project, 1959-1966

\begin{tabular}{|c|c|c|c|c|}
\hline Cervical Dilation $(\mathrm{cm})$ & $\begin{array}{l}\text { Admitted at } 2 \\
\text { or } 2.5 \mathrm{~cm}\end{array}$ & $\begin{array}{l}\text { Admitted at } 3 \\
\text { or } 3.5 \mathrm{~cm}\end{array}$ & $\begin{array}{l}\text { Admitted at } 4 \\
\text { or } 4.5 \mathrm{~cm}\end{array}$ & $\begin{array}{c}\text { Admitted at } 5 \\
\text { or } 5.5 \mathrm{~cm}\end{array}$ \\
\hline Adm. to 3 & $1.0(8.5)$ & & & \\
\hline Adm. to 4 & $2.3(12.6)$ & $0.7(6.5)$ & & \\
\hline Adm. to 5 & $3.6(15.3)$ & $1.9(10.5)$ & $0.5(5.4)$ & \\
\hline Adm. to 6 & $4.5(17.0)$ & $2.7(12.4)$ & $1.3(8.4)$ & $0.4(3.4)$ \\
\hline Adm. to 7 & $5.1(18.0)$ & $3.3(13.3)$ & $2.0(10.1)$ & $0.9(5.5)$ \\
\hline Adm. to 8 & $5.5(18.8)$ & $3.7(14.1)$ & $2.4(11.4)$ & $1.3(6.6)$ \\
\hline Adm. to 9 & $5.9(19.8)$ & $4.1(15.2)$ & $2.8(12.7)$ & $1.7(8.2)$ \\
\hline Adm. to 10 & $6.3(20.7)$ & $4.5(16.2)$ & $3.2(14.1)$ & $2.1(9.3)$ \\
\hline
\end{tabular}

Adm., admission.

Data are median (95th percentile).

multiparas was more obvious and emerged much sooner than that of the nulliparas. After the inflection point, the cervix dilated precipitously to $10 \mathrm{~cm}$. The inflection point appears slightly sooner in parity $2+$ than in parity 1 . On the other hand, the nulliparas had a quite different average labor curve. The inflection point was unclear and appeared quite late in labor.

Interpretation of the average labor curve requires caution. First, duration of labor expressed in the curves is the mean rather than the median. The mean and median may not match. For example, the duration of labor from 4 to $10 \mathrm{~cm}$ was 4.4 hours on the nulliparous labor curve (the mean) but 3.7 hours according to traverse time (the median). Furthermore, the shape of the average labor curve is determined by the shape and variability of the underlying individual curves. For instance, if parturients have diverse speeds of cervical dilation in the latent and active phases and enter the active phase at various time (measured by cervical dilation), the average labor curve will tend to look flat and the inflection point will be unclear. From the average labor curves (Fig. 1), one may deduce that the variation of multiparous curves is smaller than that of nulliparous curves; and women of parity $2+$ may enter the active phase of labor slightly sooner than parity 1 . The speed of cervical dilation in active phase is slower in nulliparas than in multiparas.

More importantly, the inflection point on the multiparous curve is at $5 \mathrm{~cm}$ or $5.5 \mathrm{~cm}$. Although we cannot be certain that all multiparas enter the active phase at $5 \mathrm{~cm}$, we may acknowledge that by $5 \mathrm{~cm}$, the vast majority of multiparous women have entered the active phase. Further, if the latest starting point of the active labor is $5 \mathrm{~cm}$ for multiparas, the inflection point for nulliparas is likely to be later than $5 \mathrm{~cm}$.

Peisner and Rosen ${ }^{15}$ examined 1,699 labors with approximately two thirds nulliparas and one third multiparas. The transition from the latent to active phase of labor was defined as time when the cervix dilated faster than $1.2 \mathrm{~cm} / \mathrm{h}$ in nulliparas and 1.5 $\mathrm{cm} / \mathrm{h}$ in multiparas. They found that among women who had no active phase arrest, $50 \%$ of them entered active phase by $4 \mathrm{~cm}$ dilation; $74 \%$ by $5 \mathrm{~cm}$ and $89 \%$ by $6 \mathrm{~cm}$. Unfortunately, mixing women with various parities makes the application of their findings to nulliparas as well as multiparas difficult.

These observations raise an important question: how should the active phase be defined? Hendricks et $\mathrm{al}^{16}$ define it as "Pains plus Progress," "which usually means that contractions are coming at intervals of five minutes or less, are associated with discomfort for the patient, and with observable progress in cervical dilatation." Based on the Friedman curve in primigravid, ${ }^{13,17}$ the transition from the latent to active phase appeared to occur between 3 and $4 \mathrm{~cm}$. A more commonly used definition is that the active phase starts at $4 \mathrm{~cm}$ dilation. ${ }^{18,19}$ However, if the observations by Peisner and Rosen ${ }^{15}$ and our study are true, there are a large number of women who are not in the active phase at $4 \mathrm{~cm}$, particularly in nulliparas. As Peisner and Rosen ${ }^{15}$ conclude, "a patient who is not progressing in labor at $4 \mathrm{~cm}$ cervical dilation is not necessarily abnormal." Our analysis shows that it may take more than 4 hours for nulliparas to progress from 4 to $5 \mathrm{~cm}$ (Table 2).

Rouse et a ${ }^{20,21}$ subsequently proposed a new definition of the active phase $(4-\mathrm{cm}$ dilatation and at least $90 \%$ effacement or 5 -cm dilatation regardless of effacement) and asserted that this definition would capture the majority of women who have entered the active phase during labor induction. ${ }^{20}$ It is yet to be tested whether this definition applies to spontaneous labor and whether it works equally well in nulliparas and multiparas.

Another important difference between Friedman curve $^{13}$ and ours is the deceleration phase. Our study as well as previous studies ${ }^{16}$ do not show a clear deceleration phase in late active phase although we used an 
eighth-degree polynomial model with a very large sample size. After examining a large number of individual labor curves, we agree with Hendricks et al ${ }^{16}$ that "the deceleration phase" may be an artifact of missed observation in most cases. As the cervical dilation accelerates when it approaches $10 \mathrm{~cm}$, prompt and frequent pelvic exams are necessary to observe for the first time when the cervix reaches the $10 \mathrm{~cm}$. If the full dilation is not observed promptly, for example, a cervical examination was not done until half an hour after the cervix had reached the full dilation, it would appear on the labor curve that the cervix had taken a long time to dilate from $9 \mathrm{~cm}$ to $10 \mathrm{~cm}$ - a deceleration phase.

Despite the fact that the average labor curve is easy to understand and provides ample information for studying labor progression, its clinical utility in managing individual patients is limited. We, therefore, propose alternative tools for clinical purposes. Table 2 presents the median and 95th percentiles of duration of labor from one dilation level to the next in women who reached full dilation and had a normal perinatal outcome. This information gives clinicians a more objective assessment of whether labor arrest has occurred. This table suggests that a 2-hour threshold ${ }^{14}$ may be too short before $6 \mathrm{~cm}$ whereas a 4 -hour limit ${ }^{22}$ may be too long after $6 \mathrm{~cm}$. Given that the speed of cervical dilation is not constant, a graduated threshold based on the level of cervical dilation may be a more appropriate approach to defining labor arrest than a "one-size-fits-all" method. Similarly, Table 3 illustrates the cumulative duration of labor from admission. It provides some guidance to diagnose labor protraction or dystocia.

It should be pointed out that, although the old data have advantages in studying natural history, ie, the shape of the labor curve and pattern of labor progression, it is necessary to exercise caution in generalizing the absolute values in Tables 2 and 3 to contemporary obstetric populations. This is because the characteristics of the parturients (eg, maternal age, body mass and fetal size) have changed substantially; so has the obstetric practice. Contemporary data, therefore, are needed to establish such references for current practice.

In summary, our observation of a large group of parturients with fewer obstetric interventions suggests that multiparas may not enter the active phase of labor until $5 \mathrm{~cm}$. Nulliparas may start the active phase even later and may not necessarily have a clear active phase characterized by precipitous dilation. The deceleration phase in the late active phase of labor may be an artifact in many cases. A graduated approach for assessing labor progression in individual patients is proposed.

\section{REFERENCES}

1. Betrán AP, Merialdi M, Lauer JA, Bing-Shun W, Thomas J, Van Look P, et al. Rates of caesarean section: analysis of global, regional and national estimates. Paediatr Perinat Epidemiol 2007;21:98-113.

2. Villar J, Valladares E, Wojdyla D, Zavaleta N, Carroli G, Velazco A, et al. Caesarean delivery rates and pregnancy outcomes: the 2005 WHO global survey on maternal and perinatal health in Latin America. Lancet 2006;367:1819-29.

3. Coleman VH, Lawrence H, Schulkin J. Rising cesarean delivery rates: the impact of cesarean delivery on maternal request. Obstet Gynecol Surv 2009;64:115-9.

4. Niswander KR, Gordon M, editors. The collaborative perinatal study of the National Institute of Neurological Diseases and Stroke: the women and their pregnancies. Philadelphia (PA): WB Saunders; 1972.

5. Friedman EA, Neff RK. Labor and delivery: impact on offspring. Littleton (MA): PSG Publishing Co., Inc.; 1987. p $9-34$.

6. Hardy JB. The Collaborative Perinatal Project: lessons and legacy. Ann Epidemiol 2003;13:303-11.

7. Crowder KJ, Hand DJ. Analysis of repeated measures. New York (NY): Chapman and Hall; 1990.

8. Klein JP, Moeschberger ML. Survival analysis: techniques for censored and truncated data. Berlin: Springer; 1997.

9. Vahratian A, Troendle JF, Siega-Riz AM, Zhang J. Methodological challenges in studying labour progression in contemporary practice. Paediatr Perinat Epidemiol 2006;20:72-8.

10. Bellamy SL, Li Y, Ryan LM, Lipsitz S, Canner MJ, Wright R. Analysis of clustered and interval censored data from a community-based study in asthma. Statist Med 2004;23:3607-21.

11. Myrianthopoulos NC, French KS. An application of the U.S. Bureau of the Census socioeconomic index to a large, diversified patient population. Soc Sci Med 1968;2:283-99.

12. Friedman EA. The graphic analysis of labor. Am J Obstet Gynecol 1954;68:1568-75.

13. Friedman EA. Primigravid labor: a graphicostatistical analysis. Obstet Gynecol 1955;6:567-89.

14. Friedman EA. Labor: clinical evaluation and management. 2nd ed. New York (NY): Appleton-Century-Crofts; 1978.

15. Peisner DB, Rosen MG. Transition from latent to active labor. Obstet Gynecol 1986;68:448-51.

16. Hendricks $\mathrm{CH}$, Brenner WE, Kraus G. Normal cervical dilatation pattern in late pregnancy and labor. Am J Obstet Gynecol 1970;106:1065-82.

17. Bowes Jr WA, Thorp Jr JM. Clinical aspects of normal and abnormal labor. In: Creasy RK, Resnik R, Iams JD, editors. Maternal-fetal medicine 5th ed. Philadelphia (PA): Saunders. 2004:671.

18. Albers LL, Schiff M, Gorwoda JG. The length of active labor in normal pregnancies. Obstet Gynecol 1996;87:355-9.

19. Rouse DJ, Owen J, Hauth JC. Active-phase labor arrest: oxytocin augmentation for at least 4 hours. Obstet Gynecol 1999;93:323-8.

20. Lin MG, Rouse DJ. What is a failed labor induction? Clin Obstet Gynecol 2006;49:585-93.

21. Rouse DJ, Owen J, Hauth JC. Criteria for failed labor induction: prospective evaluation of a standardized protocol. Obstet Gynecol 2000;96:671-7.

22. Rouse DJ, Owen J, Savage KG, Hauth JC. Active phase labor arrest: revisiting the 2-hour minimum. Obstet Gynecol 2001;98:550-4. 